\title{
Gamma-Ray Bursts from Extragalactic Magnetar Flares
}

\author{
Robert C. Duncan \\ Dept. of Astronomy, University of Texas, Austin, Texas 78712
}

\begin{abstract}
.
The prototype for events that we call MFs - "March Fifth" events or "Magnetar Flares" - was observed on March 5, 1979. There is evidence that MFs are powered by catastrophic magnetic instabilities in ultra-magnetized neutron stars. These events begin with brief ( $\Delta t \sim 0.1-1 \mathrm{~s})$, intense, hard spikes of gamma rays, probably emitted in concurrence with relativistic outflows; followed by long $(t \sim 100 \mathrm{~s})$ softer tails of hard X-rays, modulated on the stellar rotation period. Prototypical MFs could have been detected by BATSE out to $\sim 13 \mathrm{Mpc}$, nearly reaching the Virgo cluster. The likely number of isotropic, standard-candle MFs detected by the BATSE experiment is $\sim 12$. These short-duration, fast-rising gamma-ray bursts could in principle be identified by their positional coincidences with nearby galaxies and the Supergalactic Plane. The ensuing soft tail emission would not have been detected by BATSE for sources more distant than the Andromeda Galaxy. Bayes' Theorem implies that there is a $~ 99 \%$ chance for at least 1 isotropic MF in the BATSE catalog, and a $\sim 16 \%$ chance for more than 20 .

It is possible that MFs also emit an intense, hard, beamed component during the intial spike phase. If this beamed component has opening angle $\psi=8^{\circ} \psi_{8}$ and peak luminosity comparable to the power of the isotropic component, then BATSE would detect such beamed sources out to redshift $z \sim 0.1 \psi_{8}^{-1}$, at a full-sky rate of $\dot{N}_{B} \sim 100 \psi_{8}^{-1} \mathrm{yr}^{-1}$. We speculate that such beamed MFs could account for the short, hard Class II gamma-ray bursts (GRBs) in the BATSE catalog, or some significant subset of them. If true, then Class II GRBs positions should correlate with the positions of galaxies and galaxy clusters within $\sim 350 \mathrm{Mpc}$.
\end{abstract}

\section{INTRODUCTION}

The short-duration, hard-spectrum gamma-ray bursts (GRBs) in the BATSE catalog, often called Class II bursts, have no detected afterglows. As a consequence, they have not yet been subject to scrutiny for absorption lines, and the distance from Earth at which these bursts originate is uncertain. Here we will explore the possibility of a relatively nearby extragalactic origin, $z \lesssim 0.1$, for (at least) some Class II GRBs.

Kouveliotou et al. first showed that the GRB population is bimodal [1]. More 
recently, Murkherjee et al. found evidence that there exist three classes of BATSE GRBs [2]. But Hakkilla et al. (ref. [3]) argued that Class III properties can be produced from Class I by a combination of measurement error, hardness-intensity correlation, and a newly-identified BATSE bias, the fluence duration bias. Class II GRBs, which are short-duration, low-fluence, hard-spectrum events, do not seem to be related to Class I/III. It is Class II GRBs, or some subset of Class II, that we suggest are MFs.

Class II GRBs have many features in common with the hard spikes of MFs. About $40 \%$ of Class II bursts are single-peaked [4]. Most peaks exhibit a fast rise and slower decline, with hard-to-soft spectral evolution [4]. These properties are shared by the hard spikes of the March 5th and August 27th events [5-7], which also had similar spectral hardness $[8,9]$. Class II GRBs that are sufficiently bright to study with fine time resolution often show substructure down to time scales of $\sim 10 \mathrm{~ms}$ and less [10,11], as did the 1979 March 5 event [12].

\section{BATSE DETECTION OF MF'S}

Consider a beamed source of gamma-rays, emitting uniformly into a beaming fraction $f=\Delta \Omega / 4 \pi$, of the unit sphere, with peak beam luminosity $L_{\mathrm{bm}}$. For a BATSE peak-flux detection threshold $F_{B}$, the sampling depth of BATSE is

$$
D=\left(\frac{L_{\mathrm{bm}}}{4 \pi f F_{B}}\right)^{1 / 2}
$$

If $D$ is small enough that source density evolution and departures from Euclidean geometry are negligible, then BATSE's rate of event detection is

$$
\dot{N}_{B}=\frac{4 \pi}{3} D^{3} n_{*} f \Gamma
$$

Here $n_{*}$ is the density of $L_{*}$ galaxies (with luminosities comparable to that of the Milky Way) and $\Gamma$ is the rate of MFs within each $L_{*}$ galaxy. Thus the (full-sky) BATSE detection rate is

$$
\dot{N}_{B}=\frac{n_{*} \Gamma}{6(\pi f)^{1 / 2}}\left(\frac{L_{\mathrm{bm}}}{F_{B}}\right)^{3 / 2} .
$$

We evaluate this using known parameter values. In particular, $F_{B} \approx 10^{-7} \mathrm{erg}$ $\mathrm{cm}^{-2} \mathrm{~s}^{-1}$ (ref. [10]) and $n_{*}=0.01 h^{3} \mathrm{Mpc}^{-3}$ from the local normalization of the Schechter luminosity function (e.g., ref. [13]). We will adopt a Hubble constant of $H_{o}=65 h_{65} \mathrm{~km} \mathrm{~s}^{-1} \mathrm{Mpc}^{-1}$, thus $n_{*}=2.8 \times 10^{-3} h_{65}^{3} \mathrm{Mpc}^{-3}$.

Given 2 MFs in our Galaxy (or in nearby dwarf satellite galaxies) in the past $t_{o} \sim 20$ years of effective full sky coverage during which have had capability for detecting them (Hurley 1999, private communication), the MF rate per $L_{*}$ galaxy is roughly 


$$
\Gamma=0.1 \Gamma_{0.1} \mathrm{yr}^{-1} .
$$

The simplest idealization is that the gamma-rays in MF hard spikes are emitted isotropically, $f \approx 1$. Since the long, soft, oscillating tails of MFs have never been observed without hard spikes at their onsets, we infer that many or most MFs begin with a spike of quasi-isotropic emissions. (This is in addition to a possible highintensity, beamed component. We have no direct evidence for beamed emission; however, with only 2 detected MFs, we would not expect to have observed any beams with $f \ll 1$.)

Setting $f=1$ in the above formulae, we find a BATSE sampling depth for the isotropic component of MFs of

$$
D=13\left(L_{45} / 2\right)^{1 / 2} \mathrm{Mpc},
$$

where we have scaled to the value of peak luminosity found for the 1979 March 5 event by Fenimore [8]. This falls just short of the Virgo cluster at $D$ [virgo] $\approx 18 h_{65}^{-1}$ Mpc. The full-sky BATSE detection rate is

$$
\dot{N}_{B}=2.6\left(L_{45} / 2\right)^{3 / 2} \Gamma_{10} h_{65}^{3} \mathrm{yr}^{-1}
$$

Since BATSE operated for 9.5 years, corresponding to 4.75 yrs of full-sky coverage, there should be

$$
\langle\mathcal{N}\rangle \sim 12\left(L_{45} / 2\right)^{3 / 2} \Gamma_{0.1} h_{65}^{3}
$$

extragalactic MFs in the BATSE catalog, detected via their quasi-isotropic hard spike emissions.

The most uncertain parameter in this estimate is $\Gamma$, the galactic rate of MFs. The Bayesian probability distribution for $\Gamma, \mathcal{P} \equiv d P / d \Gamma$, is

$$
\mathcal{P}(\Gamma)=\frac{P(2 \mid \Gamma) \mathcal{P}_{\text {prior }}(\Gamma)}{\int_{0}^{\infty} d \Gamma P(2 \mid \Gamma) \mathcal{P}_{\text {prior }}(\Gamma)},
$$

where the probability for observing $2 \mathrm{MFs}$ in time $t_{o} \approx 20 \mathrm{yr}$, given $\Gamma$, is $P(2 \mid \Gamma)=\frac{1}{2}\left(\Gamma t_{o}\right)^{2} \exp \left(-\Gamma t_{o}\right)$ since this is a Poisson process. The appropriate prior distribution, when we don't know the order of magnitude of $\Gamma$ a priori, is $\left(d P_{\text {prior }} / d \log \Gamma\right)=$ constant, or $\mathcal{P}_{\text {prior }} \propto \Gamma^{-1}$. Thus

$$
\mathcal{P}(\Gamma)=\Gamma t_{o}^{2} \exp \left(-\Gamma t_{o}\right),
$$

with a mean value $\langle\Gamma\rangle=2 t_{o}^{-1}=0.1 \mathrm{yr}^{-1}$ as noted above [eq. (4)]. The probability for the galactic flare rate to exceed a cutoff value, $\Gamma>\Gamma_{x}$, is thus $P\left(\Gamma>\Gamma_{x}\right)=\exp \left(-\Gamma_{x} t_{o}\right)\left(1+\Gamma_{x} t_{o}\right)$. If other sources of uncertainty can be neglected, then there is a $99 \%$ probability for at least one isotropic MF in the BATSE catalog, $\mathcal{N}>1$. The probability is $80 \%$ for $\mathcal{N}>5 ; 52 \%$ for $\mathcal{N}>10 ; 16 \%$ for $\mathcal{N}>20 ;$ and $4.5 \%$ for $\mathcal{N}>30$. 


\section{COULD ALL CLASS II GRB'S BE MF'S?}

Suppose that there also exists a beamed component in the intial hard spikes of MFs, with a full opening angle $\psi$. The beaming fraction is $f=[1-\cos (\psi / 2)]$ assuming two beams, one at each magnetic pole. This is $f \approx \psi^{2} / 8$ for $\psi \ll 1$, or $f=2 \times 10^{-3} \psi_{8}^{2}$, where $\psi_{8} \equiv\left(\psi / 8^{\circ}\right)$. From eqs. (1)-(3), the BATSE sampling depth for beamed MFs is

$$
D=350 \psi_{8}^{-1}\left(\frac{L_{\mathrm{bm}}}{3 \times 10^{45} \mathrm{erg} \mathrm{s}^{-1}}\right)^{1 / 2} \mathrm{Mpc}
$$

and the (full-sky) rate of detection is

$$
\dot{N}_{B}=100 \Gamma_{0.1} h_{65}^{3} \psi_{8}^{-1}\left(\frac{L_{\mathrm{bm}}}{3 \times 10^{45} \mathrm{erg} \mathrm{s}^{-1}}\right)^{3 / 2} \mathrm{yr}^{-1}
$$

This is plausibly in agreement with the rate of detection of Class II bursts by BATSE. Out of 796 bursts classified by Murkherjee et al., about 185 were Class II, or $\sim 23 \%$. Since BATSE detects about 300 GRB per year with half-sky coverage (due to the fact that the Compton Observatory is in low Earth orbit), the full-sky detection rate of Class II bursts is roughly $\dot{N}_{B}$ (Class II) $\approx 140 \mathrm{yr}^{-1}$.

However, there is no compelling reason to expect such a beamed emission component based upon the magnetar model [14-16,7].

Moreover, the number-intensity or $V / V_{\max }$ distribution of Class II GRBs seems to give evidence against a local extragalactic origin for these events. This is a serious concern; however, note that the evidence for $\left\langle V / V_{\max }\right\rangle<0.5$ in Class II bursts is much less compelling than in Class I/III. It is possible that selection effects cause a paucity of BATSE events just above threshold for peak flux on 256 and 64-ms time scales. (Flux averaged over $1.024 \mathrm{~s}$ does not give a good brightness measure for these short bursts.) Faint and poorly-measured bursts are systematically removed because of insufficient information to make class identifications (e.g., only 778 out of 1122 catalogued bursts were classified in ref. [3]); and the class identifications themselves are least reliable near threshold where measurements are most statistically dubious. It is also possible that the bright Class II bursts include a contamination of physically-distinct, non-Euclidean (low $V / V_{\max }$ ) events; e.g., a tail of Class I bursts extending to short durations, or a subclass of short bursts from galactic SGRs or AXPs like the bright, hard-spectrum events with $T_{90} \sim 1 \mathrm{~s}$ already identified from SGR 1900+14 [17]. Note that Tavani [18] found $\left\langle V / V_{\max }\right\rangle=0.458 \pm 0.044$ for short duration, soft spectrum bursts in the BATSE catalog (66 events with $T_{90}<2.5 \mathrm{~s}$ and $\left.H_{32}^{e}<3\right)$, and Cline, Matthey \& Otwinowski [19] found $\left\langle V / V_{\max }\right\rangle=0.52 \pm 0.06$ for all BATSE bursts with $T_{90}<0.1 \mathrm{~s}$. 


\section{CONCLUSIONS: OBSERVATIONAL TESTS}

Under the idealization that MF hard spikes are emitted quasi-isotropically, we find that the BATSE catalog contains $\sim 12$ extragalactic MFs [eq. (7)]. These events are expected to be fast-rising $\left(t_{\text {rise }} \lesssim 1 \mathrm{~ms}\right)$, short-duration bursts, correlated in position with galaxies at $D_{\text {gal }}<20 \mathrm{Mpc}$. Insofar as the quasi-isotropic component of MF hard spikes have uniform peak luminosities, there will be a diminishment of the peak flux with $D_{\text {gal }}$ according to $F_{\text {peak }} \simeq L_{\text {iso }} /\left(4 \pi D_{\text {gal }}^{2}\right)$, affording a possible auxiliary check on associations. As a group, these "isotropic MFs" should tend to concentrate toward the supergalactic plane [20]. Hartmann, Briggs \& Mannheim (ref. [21]) found no significant supergalactic anisotropy in the BATSE catalog, but their statistics were dominated by Class I bursts. At distances less than $D_{\text {vir }} \sim 18 h_{65}^{-1} \mathrm{Mpc}$ the distribution of candidate MFs may show a significant Virgocentric dipole moment (e.g., Fig. 3.3 in ref. [13]); and a correlation with Virgo's discrete position on the sky if the sampling depth (eq. [5]) extends as far as $D_{\text {vir }}$.

This prediction of "isotropic MFs" in the BATSE catalog is based upon direct, reliable extrapolation from observations of the 1979 March 5 and 1998 August 27 events. More speculatively, in any model of MFs, a full-sky BATSE rate $\dot{N}_{B}$ is possible if there exists a beamed emission component with full opening angle

$$
\psi=8^{\circ} \Gamma_{0.1} h_{65}^{3}\left(\frac{L_{\mathrm{bm}}}{3 \times 10^{45} \mathrm{erg} \mathrm{s}^{-1}}\right)^{3 / 2}\left(\frac{\dot{N}_{B}}{100 \mathrm{yr}^{-1}}\right)^{-1},
$$

where $L_{\mathrm{bm}}$ is the total beam peak power. This equation assumes two polar beams; for a single beam, $\psi$ goes up by $\sqrt{2}$.

If such beamed MFs accounted for many or all Class II GRBs, then the events would have positions correlated with galaxies and galaxy clusters within the BATSE sampling depth at redshift

$$
z_{\mathrm{B}}=0.076 \psi_{8}^{-1} h_{65}\left(\frac{L_{\mathrm{bm}}}{3 \times 10^{45} \mathrm{erg} \mathrm{s}^{-1}}\right)^{1 / 2} .
$$

Several studies have found correlations of GRB positions with galaxy clusters in the Abell, Corwin \& Olowin (hereafter ACO) catalog [22], but only at a statistically marginal level [23-25]. These studies have focused primarily on bursts with small positional error boxes, which are mostly bright Class I events. Note that the MFs come from young neutron stars in star-forming regions. Such sources are not expected to concentrate strongly within rich galaxy clusters, which contain mostly early-type (gas-stripped) galaxies.

The positional correlations expected for MFs are difficult to study using BATSE data. Class II GRBs, with short durations and relatively low fluences, tend to have poorly-determined positions, often with BATSE error boxes of size $\sim 10^{\circ}$. Future experiments will localize short-duration GRBs well enough to test for correlations with nearby galaxies and galaxy clusters, hopefully making the identification of extragalactic MFs possible. 


\section{ACKNOWLEDGMENTS}

We thank R. Knill-Dgani for discussions. This work was supported by NASA grant NAG5-8381; by the Texas Advanced Research Program grant ARP-028.

\section{REFERENCES}

1. Kouveliotou, C. et al., Ap.J., 413, L101 (1993).

2. Murkherjee, S. et al., Ap.J., 508, 314 (1998).

3. Hakkila, J. et al., Ap.J, 538, 165 (2000).

4. Gupta, V., Gupta, P.D., and Bhat, P.N. in Gamma-Ray Bursts: 5th Huntsville Symposium, eds. R.M. Kippen et al., New York: AIP Conf. Proc. No. 526, pp. 215219 (2000).

5. Mazets, E.P. et al., Nature, 282, 587 (1979).

6. Mazets, E.P. et al., Astron. Lett., 25 (10), 635 (1999).

7. Feroci, M., Hurley, K., Duncan, R.C. and Thompson, C., Ap J, 549, in press (2001) [astro-ph / 0010494].

8. Fenimore, E., in High-Velocity Neutron Stars and Gamma-Ray Bursts, ed. R.E. Rothschild \& R.E. Lingenfelter, New York: AIP Conf. Proc. No. , pp. 68-72 (1996).

9. Fenimore, E., Klebesadel, R. and Laros, J., Ap.J., 460, 964 (1996).

10. Meegan, C. et al., ApJ Supp., 92, 229 (1994).

11. Meegan, C. et al., ApJ Supp., 106, 65 (1996).

12. Barat, C. et al., Astron. \& Astroph., 126, 400 (1983).

13. Peebles, P.J.E., Principles of Physical Cosmology, Princeton: Princeton University Press (1993).

14. Duncan, R.C. and Thompson, C., Ap.J., 392, L9 (1992).

15. Thompson, C. and Duncan, R.C., M.N.R.A.S., 275, 255 (1995).

16. Thompson, C. and Duncan, R.C., Ap.J., submitted (2001).

17. Woods, P.M. et al., Ap.J., 527, L47 (1999).

18. Tavani, M., Ap.J., 497, L21 (1998).

19. Cline, D.B., Mathhey, C. and Otwinowski, S. Ap.J., 527, 827 (1999).

20. de Vaucouleurs, G., Astronom.J., 58, 29 (1953).

21. Hartmann, D.H., Briggs, M.S. and Mannheim, K., in Gamma-Ray Bursts: 3rd Huntsville Symposium, eds. M.A. Briggs et al., New York: AIP Conf. Proc. 384, pp. 397-403 (1996).

22. Abell, G.O., Corwin, H. and Olowin, R.P., Ap.J.Supp., 70, 1 (1989).

23. Marani, G.F., Nemiroff, R.J., Norris, J.P. and Bonnell, J.P., Ap.J., 474, 576 (1997).

24. Kolatt, T. and Piran, T., Ap.J., 467, L41 (1996).

25. Hurley, K. et al., Ap.J., 479, L113 (1997). 